\title{
Reduced Polynomial Order Linear Prediction
}

\author{
Eric M. Dowling, Senior Member, IEEE, Ronald D. DeGroat, Member, IEEE, Darel A. Linebarger, \\ Senior Member, IEEE, Louis L. Scharf, Fellow, IEEE and Marvin Vis, Member, IEEE
}

\begin{abstract}
Reduced rank linear predictive frequency and direction-of-arrival (DOA) estimation algorithms use the singular value decomposition (SVD) to produce a noise-cleaned linear prediction vector. These algorithms then root this vector to obtain a subset of roots, whose angles contain the desired frequency or DOA information. The roots closest to the unit circle are deemed to be the "signal roots." The rest of the roots are "extraneous." The extraneous roots are expensive to calculate. Further, a search must be done to discern the signal roots from the extraneous roots. Here, we present a reduced polynomial order linear prediction method that simplifies the rooting computation for applications where high-speed processing is critical.
\end{abstract}

\section{INTRODUCTION}

$\mathbf{T}$ HE Prony method roots a linear prediction (LP) polynomial to find the parameters of an exponential data model [1]. While nonlinear least squares or maximum likelihood are the off-line exponential model parameter estimators of choice, Prony's method has found widespread use in signal processing due to its computational efficiency and its suitability to realtime implementation. The Prony method has the drawback that errors in the linear prediction vector can propagate dramatically to errors in the root locations. In the past, reduced rank Prony estimators have been proposed to increase performance. These estimators include reduced rank least squares (LS) linear prediction [2], the minimum norm method [3], the total least (TLS) squares method [4], some constrained TLS approaches [5], and others. For LP-based frequency or DOA estimation, it is generally agreed that reduced rank TLS outperforms reduced rank LS. Further, the minimum norm method is equivalent to the reduced rank TLS method [6]. Therefore, this letter focuses on minimum norm, i.e., reduced rank TLS based estimators.

One drawback to reduced rank linear prediction is the need to root an oversized polynomial and to then extract the signal roots from the extraneous roots. For example, suppose $r=2$ signal directions are to be estimated from an $m=50$ linearly equally spaced (LES) sensor array. After the prediction-error vector is computed, an order 50 polynomial must be rooted, and then, a search must be performed to find the $r=2$ roots closest to the unit circle out of the set of 50 computed roots. Here, we propose a reduced rank linear prediction method that

Manuscript received May 19, 1995. This work was supported, in part, by the National Science Foundation Grant MIP-9203296 and the Texas Advanced Research Program Grant 009741-022. The associate editor coordinating the review of this letter and approving it for publication was Prof. T. S. Durrani.

E. M. Dowling, R. D. DeGroat, and D. A. Linebarger are with the Erik Jonsson School of Engineering and Computer Science, The University of Texas at Dallas, Richardson, TX 75083-0688 USA (e-mail: emd@utdallas.edu).

L. L. Scharf and M. Vis are with the University of Colorado, Boulder, CO USA.

Publisher Item Identifier S 1070-9908(96)01832-9. only requires an order $r$ polynomial to be rooted. The $r$ roots of this polynomial are the desired signal roots.

It is important to note that matrix pencil-oriented DOA estimators have been designed to eliminate extraneous roots [7]-[10]. Those schemes perform an SVD, then another SVD, and produce an unsymmetric eigenproblem whose $r$ eigenvalues are the desired $r$ roots. The proposed method is similar in that two SVD's are required, followed by an order $r$ polynomial rooting. Therefore, this note brings about a polynomial order reduction for the linear prediction techniques similar to those available in the pencil methods. This is especially important in reducing the rooting computation in subspace-based frequency and DOA tracking problems [11].

The rest of this letter is organized as follows. In Section II, we present the data model used throughout. This data model will assume spatial sensor data; however, a similar discussion could be given in terms of an exponential time series model. In Section III, we introduce the root-basis for the noise subspace. In Section IV, we derive the reduced rank and polynomial order linear predictive DOA estimator. In Section V, we analyze performance, and in Section VI, we offer conclusions.

\section{The Data Model.}

Assume that $r$ monochomatic plane waves propagating from different directions impinge on an LES array of $m$ sensors located in the same plane as the sources. The array output snapshot vectors can be modeled as

$$
\mathbf{x}(k)=\mathbf{A} \mathbf{s}(k)+\mathbf{n}(k)
$$

where $\mathbf{A}=\left[\mathbf{a}\left(\theta_{1}\right)|\cdots| \mathbf{a}\left(\theta_{r}\right)\right]_{m \times r}$ is a matrix of linearly independent source direction vectors, $\mathbf{s}(k)=e^{j \omega k}\left[s_{1}(k), \ldots\right.$, $\left.s_{r}(k)\right]^{T}$ is the $r \times 1$ signal modulation vector whose elements may be complex random variables, $\omega$ is the temporal frequency of the propagating narrow-band plane waves, and the elements of the noise vector $\mathbf{n}(k)=\left[n_{1}(k), \ldots, n_{m}(k)\right]^{T}$ consist of zero mean, white, complex Gaussian noise with variance $\sigma^{2}$. In addition, assume that the signal and noise are uncorrelated with each other. The direction vectors are defined by the array manifold $\mathbf{a}(\theta)=\left[1, e^{j \theta}, e^{j 2 \theta}, \ldots, e^{j(m-1) \theta}\right]^{T}$, where $\theta=\pi d \sin \phi$, and $\phi$ is the DOA measured with respect to broadside. Here, $\theta$ is the so-called "electrical" angle, and $d$ is the sensor spacing in units of half wavelengths. With this data model, the correlation matrix takes the form

$$
\mathbf{R}=E\left[\mathbf{x}(k) \mathbf{x}^{H}(k)\right]=\mathbf{A} \mathbf{P} \mathbf{A}^{H}+\sigma_{n}^{2} \mathbf{I}
$$

where $\mathbf{P}=E\left[\mathbf{s}(k) \mathbf{s}^{H}(k)\right]$ is the $r \times r$ signal correlation matrix, and $E[\cdot]$ is the expectation operator. If we also assume 
that $\mathbf{P}$ is full rank, the eigendecomposition of $\mathbf{R}$ can be partitioned as

$$
\mathbf{R}=\left[\begin{array}{ll}
\mathbf{V}_{s} & \mathbf{V}_{n}
\end{array}\right]\left[\begin{array}{cc}
\Lambda_{s} & 0 \\
0 & \sigma_{n}^{2} \mathbf{I}_{m-r}
\end{array}\right]\left[\begin{array}{ll}
\mathbf{V}_{s} & \mathbf{V}_{n}
\end{array}\right]^{H}
$$

where $\mathbf{V}_{s}$ spans the signal subspace $(\operatorname{Range}(\mathbf{A}))$, and $\mathbf{V}_{n}$ spans the noise subspace. In the algorithm we develop, we will never need to form the correlation matrix.

\section{The RoOt-Basis For the NoISE SubSPACE}

Let $\alpha(z)$ be the transfer function of a complex FIR filter given by

$$
\alpha(z)=1+\alpha_{1} z^{-1}+\cdots \alpha_{r} z^{-r} .
$$

We can think of this filter as a spatial frequency filter. Here, the $z^{-1}$ terms represent a spatial shift operator of one sensor. We construct $\alpha(z)$ so that its roots are the signal frequencies, that is

$$
\left.\alpha(z)\right|_{z \in\left\{e^{\left.j \theta_{1}, \ldots, e^{j \theta_{r}}\right\}}\right.}=0 .
$$

With this construction, the filter produces a zero output whenever it is swept across the signal vectors. That is [5], [12]

$$
\left[\begin{array}{c}
\mathbf{a}^{H}\left(\theta_{1}\right) \\
\vdots \\
\mathbf{a}^{H}\left(\theta_{r}\right)
\end{array}\right]\left[\begin{array}{ccc}
1 & & \\
\alpha_{1}^{*} & \ddots & \\
& \ddots & 1 \\
\alpha_{r}^{*} & & \alpha_{1}^{*} \\
& \ddots & \\
& & \alpha_{r}^{*}
\end{array}\right]=\mathbf{0}_{r \times m-r}
$$

or, in compact matrix notation

$$
\mathbf{A}^{H} \mathbf{N}=\mathbf{0}_{\boldsymbol{m}-r \times m-r} .
$$

Here, $\mathbf{N} \in \mathbb{C}^{m \times(m-r)}$ is the Toeplitz matrix of filter coefficients as given in (6). Note that by construction, the columns of $\mathrm{N}$ are linearly independent since its staggered structure prevents any linear combination of columns from annihilating any other. In addition, from (6), we can see that the $m-r$ columns of $\mathbf{N}$ are orthogonal to the $\operatorname{Range}(\mathbf{A})$, which is the signal subspace. Hence, the columns of $\mathbf{N}$ form a basis for the orthogonal complement of the signal subspace, i.e., they form a basis for the noise subspace. Because this basis is constructed from shifted polynomials whose roots are the signal roots, we call this the root-basis for the noise subspace.

\section{Reduced Rank AND Polynomial ORDER LINEAR PREDICTION}

Start with (7) and note that $\mathbf{V}_{s}=\mathbf{A T}$, where $\mathbf{T} \in \mathbb{C}^{r \times r}$ is some nonsingular transformation. Thus, we have $\mathbf{V}_{s}^{H} \mathbf{N}=\mathbf{0}$, or

$$
\left[\begin{array}{c}
\mathbf{v}_{1}^{H} \\
\vdots \\
\mathbf{v}_{r}^{H}
\end{array}\right]\left[\begin{array}{ccc}
1 & & \\
\alpha_{1}^{*} & \ddots & \\
& \ddots & 1 \\
\alpha_{r}^{*} & & \alpha_{1}^{*} \\
& \ddots & \\
& & \alpha_{r}^{*}
\end{array}\right]=\mathbf{0}_{r \times m-r}
$$

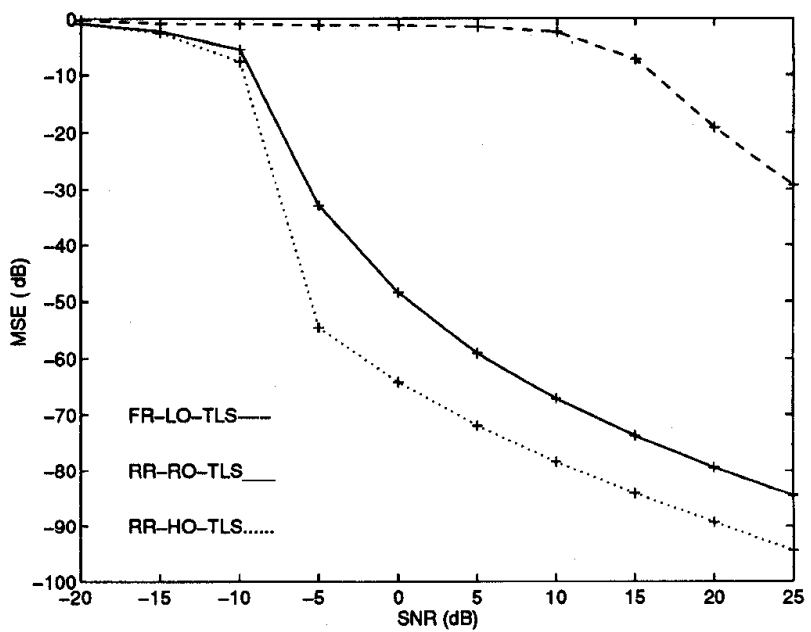

Fig. 1. Estimation mean square error (MSE) of two very closely spaced signals with $0.48 \pi \& 0.5 \pi$ normalized electrical angles as a function of SNR. Each point on the plot corresponds to a 500 point Monte Carlo simulation with 400 points of data per run.

This is an overdetermined system of $r m-r^{2}$ equations with $r$ unknowns. Note that the matrix $\mathbf{V}_{s}$ is "noise cleaned" due to the fact that its columns are orthogonal to the noise subspace. We can rearrange (8) into the standard TLS form by defining

$$
\Gamma_{i}=\left[\begin{array}{c}
\mathbf{v}_{1}^{H}(i: r+i) \\
\vdots \\
\mathbf{v}_{r}^{H}(i: r+i)
\end{array}\right] \in \mathbb{C}^{r \times(r+1)}
$$

where we use the Matlab style colon notation, that is $v_{1}^{H}(i$ : $r+i)$ is the subvector of $v_{1}^{H}$ starting at the $i$ th element and going to the $(r+i)$ th element. Next, form the matrix

$$
\Gamma=\left[\begin{array}{c}
\Gamma_{1} \\
\vdots \\
\Gamma_{m-r}
\end{array}\right] \in \mathbb{C}^{r(m-r) \times(r+1)}
$$

and solve the TLS problem

$$
\Gamma \alpha^{*}=\mathbf{0}_{m-r}
$$

where $\alpha^{*}$ is a vector whose elements, after scaling by its first element $\alpha_{0}^{*}$ are the complex conjugates of the filter coefficients given in (4). The TLS solution to this problem is the right singular vector of $\boldsymbol{\Gamma}$ corresponding to the minimum singular value, normalized by its first component. Note that $\Gamma$ is "tall and skinny" so that when we reduce $\Gamma$ to triangular form via Householder transformations, we are left with a simple $(r+1) \times(r+1)$ SVD. In addition, since only the right singular vectors are needed, the $U$ matrix of the SVD need not be accumulated. The final step is to compute the $r$ roots of $\alpha(z)$. The phase angles of these roots are the electrical angles of the signals. Hence, the $O\left(\mathrm{~m}^{2}\right)$ rooting operation is replaced with an $o\left(m r^{3}\right)$ Householder triangularization, an $O\left((r+1)^{3}\right)$ SVD, and an $O\left(r^{2}\right)$ reduced order rooting operation. Savings are significant provided $r^{3}<<m$. 


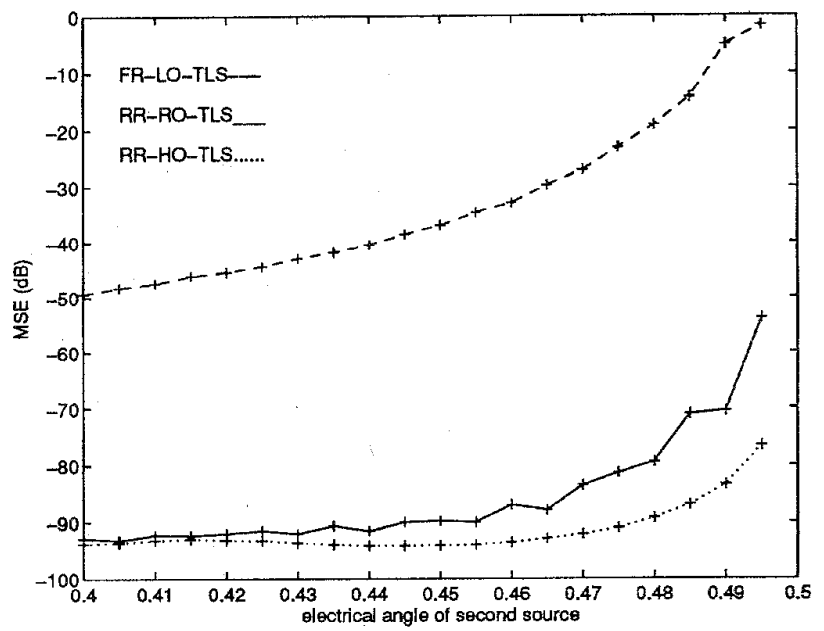

Fig. 2. Estimation mean square error (MSE) of two signals at $20 \mathrm{~dB}$ SNR when one signal has a fixed electrical angle of $.5 \pi$, and the second signal's electrical angle varies from $0.4 \pi$ to $0.5 \pi$ in steps of $0.005 \pi$ as a function of SNR. Each point on the plot corresponds to a 500 point Monte Carlo simulation with 400 points of data per run.

\section{PERformanCE ANALYSIS}

To study the performance of the reduced rank and reduced order polynomial TLS (RR-RO-TLS) algorithm, we compare its DOA estimation mean square error (MSE) to the full rank (lowest polynomial order) TLS (FR-LO-TLS) and reduced rank (highest polynomial order) TLS (RR-HO-TLS).

In the first set of simulations, $r=2$ signals with normalized electrical angles of $\theta_{1}=0.48 \pi$ and $\theta_{2}=0.5 \pi$ are to be estimated with an $m=40$ sensor, $\frac{\lambda}{2}$ spaced, LES array. Fig. 1 shows the MSE performance of the three approaches as a function of SNR. Note that FR-LO-TLS cannot resolve the signals until the SNR reaches $20 \mathrm{~dB}$ and, even then, has comparably high variance. The RR-HO-TLS and RR-RO-TLS approaches both resolve the signals at $-5 \mathrm{~dB}$ SNR. We note that RR-RO-TLS performs comparably to RR-HO-TLS but not quite as well. Therefore, we give up some performance in order to reduce the computation. In real-time applications, this tradeoff may be desirable.

The next set of simulations fixes the SNR at $20 \mathrm{~dB}$. One source is fixed to have a normalized electrical angle of $0.5 \pi$, whereas the normalized electrical angle of the second source is moved from $0.4 \pi$ to $0.495 \pi$ in increments of $0.005 \pi \mathrm{rad}$. What we see is that the MSE of the reduced rank methods are almost identical when the sources are separated by more than $0.05 \pi \mathrm{rad}$. When the sources become closely spaced, RRRO-TLS loses some accuracy but is still able to resolve the sources and continues to have comparable performance with the more expensive RR-HO-TLS. Again, both reduced rank methods significantly outperform the FR-LO-TLS. Note that the source separation in the simulation of Fig. 1 corresponds to the $0.48 \pi$ point on the curve of Fig. 2 . As we begin to separate the sources, the RR-RO-TLS and RR-HO-TLS MSE versus SNR curves merge together.

\section{CONCLUSION}

In this letter, a reduced rank and reduced polynomial order linear predictive DOA estimator is proposed. The method greatly outperforms full rank (lowest polynomial order) TLS linear prediction. The proposed method gives up some performance relative to the large polynomial approach but has comparable accuracy and resolution and can be computed in $O\left(m r+r^{3}\right)$ time as opposed to $O\left(m^{2}\right)$ or greater time for the full polynomial rooting. Hence, in real-time applications, where $r^{3} m$, the reduced rank and polynomial order approach provides accurate results at greatly reduced computation. The approach is currently being studied in the context of subspacebased DOA and frequency tracking.

\section{REFERENCES}

[1] S. L. Marple, Digital Spectral Analysis With Applications. Englewood Cliffs, NJ: Prentice-Hall, 1987.

[2] D. W. Tufts and R. Kumaresan, "Estimation of frequencies of multiple sinusoids: Making linear prediction perform like maximum likelihood," in Proc. IEEE, vol. 70, pp. 975-989, Sept. 1982.

[3] R. Kumaresan and D. W. Tufts, "Estimating the angles of arrival of multiple plane waves," IEEE Trans. Aerospace Electron. Syst., vol. AES-19, pp. 134-139, Jan. 1982.

[4] M. A. Rahman and K. Yu, "Total least squares approach for frequency estimation using linear prediction," IEEE Trans. Acoust., Speech, Signal Processing, vol. ASSP-34, no. 10, pp. 1440-1354, Oct. 1987.

[5] E. M. Dowling, R. D. DeGroat, and D. A. Linebarger, "Exponential parameter estimation in the presence of known components and noise," IEEE Trans. Antennas Propagat., vol. 42, no. 5, pp. 590-599, May 1994.

[6] E. M. Dowling and R. D. DeGroat, "The equivalence of total least squares and minimum norm methods," IEEE IEEE Trans. Signal Processing, vol. 39, no. 8, pp. 1891-1892, Aug. 1991.

[7] M. D. Zoltowski and D. Stavrinides, "Sensor array signal processing via Procrustes rotations based eigenanalysis of the ESPRIT data pencil," IEEE Trans. Acoust., Speech, Signal Processing, vol. 37, no. 6, pp. 832-861, June 1989.

[8] Y. Hua and T. K. Sarkar, "Matrix pencil method for estimating parameters of exponentially damped/undamped sinusoids in noise," IEEE Trans. Acoust., Speech, Signal Processing, vol. 38, no. 11, pp. 814-824, May 1990.

[9] R. Roy and T. Kailath, "ESPRIT-estimation of signal parameters via rotational invariance techniques," IEEE Trans. Acoust, Speech, Signal Processing, vol. 37, pp. 984-995, July 1989.

[10] B. D. Rao and K. S. Arun, "Model based processing of signals: A state space approach," in Proc. IEEE, vol. 80, no. 2, pp. 283-309, Feb. 1992.

[11] E. M. Dowling, L. P. Ammann, and R. D. DeGroat, "A TQR-iteration based SVD for real time angle and frequency tracking," IEEE Trans. Signal Processing, pp. 914-925, Apr. 1994.

[12] R. Kumaresan, L. L. Scharf, and A. K. Shaw, "An algorithm for polezero modeling and spectral analysis," IEEE Trans. Acoust., Speech, Signal Processing, vol. ASSP-34, no. 3, pp. 637-640, June 1986. 